a significant percentage of the airways obstruction (as determined by SGAW) in our patients and that this airways obstruction may be decreased by measures focused on bronchial clearance, such as physiotherapy. Additionally, our findings reinforce the value of chest physiotherapy in patients with a large volume of sputum production and airflow obstruction; objective data on its value has hitherto been sparse.

We thank all the consultant physicians at the Brompton Hospital who kindly allowed us to study patients under their care and also Miss J M Wright for secretarial help.

Dr G M Cochrane was supported by a grant from the Board of Governors of the National Heart and Chest Hospitals.

Requests for reprints should be addressed to Dr G M Cochrane.
References

${ }^{1}$ Lancet, 1965, 2, 776

2 March, H, Archives of Physical Medicine and Rehabilitation, 1971, 51, 528.

${ }^{3}$ Pham, Q T, et al, Bulletin de Physio-Pathologie Respiratoire, 1973, 9, 293.

4 Clarke, S W, Jones, J G, and Oliver, D R, Fournal of Applied Physiology, 1970, 29, 464.

5 Cotes, J E, Lung Function, 2nd edn. Oxford, Blackwell Scientific, 1968

${ }^{6}$ Cotes, J E, et al, British Medical fournal, 1966, 1, 1016.

7 Dubois, A B, et al, fournal of Clinical Investigation, 1956, 35, 322

${ }^{8}$ Dubois, A B, Botello, S Y, and Comroe, J H, jun, fournal of Clinical Investigation, 1956, 35, 327.

${ }^{9}$ Gaskell, D V, and Webber, B A, Brompton Hospital Guide to Chest Physiotherapy, 3rd edn. London, Blackwell, 1977.

10) Macklem, P T, American fournal of Medicine, 1972, 52, 721.

(Accepted 31 August 1977)

\title{
Rapid increases in plasma prostaglandin concentrations after vaginal examination and amniotomy
}

\author{
M D MITCHELL, A P F FLINT, J BIBBY, J BRUNT, J M ARNOLD, A B M ANDERSON, \\ A C TURNBULL
}

evidence has shown that parturition in most species, including primates, is associated with raised concentrations of prostaglandins in intrauterine tissues, plasma, or amniotic fluid, ${ }^{1-3}$ and this has focused attention on the possibility that prostaglandins may be released in response to disruption of intrauterine tissues such as decidua ${ }^{4}$ or fetal membranes. ${ }^{56}$

Recent work from this department ${ }^{7}$ has shown that amniotic fluid concentrations of the primary prostaglandin PGF and its major circulating metabolite 13,14-dihydro-15-ketoprostaglandin F (PGFM) are significantly higher in samples obtained by amniotomy than at amniocentesis. We suggested that this may relate to the increased disruption at amniotomy of the membranes that contain prostaglandin synthesising enzymes ${ }^{6}$ or it may be a process analagous to that found in pregnant sheep, ${ }^{8}{ }^{9}$ where marked PGF release into the uterine vein occurs within minutes of vaginal and cervical stimulation. Since amniotomy both stimulates the upper genital tract (vagina and cervix) and disrupts the membranes, and since these two procedures might theoretically stimulate increased intrauterine prostaglandin production, it became clear that we had to examine the effect of these procedures separately.

We therefore studied the effects of $(a)$ stimulating the upper genital tract (vaginal examination); (b) disrupting the attachment of part of the fetal membranes by separating them from the lower uterine segment (vaginal examination plus membrane sweep); and (c) puncturing the membranes vaginally to induce labour (amniotomy).

The availability of a sensitive and specific radio-immunoassay for PGFM allowed us to study circulating concentrations of this metabolite in the peripheral plasma in response to these procedures.

Nuffield Department of Obstetrics and Gynaecology, University of Oxford, John Radcliffe Hospital, Oxford OX3 9DU

M D MITCHELL, MA, DPHIL, medical research fellow

A P F FLINT, BSC, PHD, senior biochemist (now senior scientific officer, department of physiology, ARC Institute of Animal Physiology, Babraham, Cambridge)

J BIBBY, MB, MRCOG, clinical research assistant

J BRUNT, BSC, research assistant

J M ARNOLD, $\mathrm{MB}, \mathrm{BCH}$, senior house officer (now senior house officer, Royal United Hospital, Bath)

A B M ANDERSON, MD, PHD, lecturer

A C TURNBULL, MD, FRCOG, Nuffield professor of obstetrics and gynaecology

\section{Patients and methods}

In all patients pregnancy had progressed beyond 37 weeks of gestation from the first day of the last menstrual period. There was no difference among the groups studied in the mean length of gestation, age or parity of the patients, or cervical "ripeness." The pregnancies were considered to be uncomplicated with no major maternal or fetal pathology. All patients were subsequently delivered vaginally of single healthy live born infants, who were considered to be "mature" with 
no evidence of growth retardation at paediatric assessment. The full and informed consent of each patient was obtained.

The women were divided into three groups. The first group underwent vaginal examination at the antenatal clinic. Each patient lay supine, and two fingers were inserted into the vagina far enough to reach the cervix and assess the length, consistency, and dilatation of the cervical canal; the canal was not penetrated to any distance. Another group underwent a similar examination but this time the doctor inserted a finger as far as possible through the cervical canal and swept the fetal membranes off the lower uterine segment. In the third group of patients amniotomy was performed in the labour ward with patients in the supine position. With a finger in the cervical canal the fetal membranes were punctured by grasping and pulling with an amniohook; amniotic fluid was allowed to escape through the hole.

Blood samples were taken before these procedures were performed and again exactly five minutes later. Peak prostaglandin concentrations have been found in the circulation within five minutes of vaginal and cervical stimulation in sheep. ${ }^{8} 9$ Samples were collected into ice-cold tubes containing acetylsalicylic acid and ethylenediaminetetra-acetic acid. Plasma was separated within one hour of collection, by centrifugation at $2500 \mathrm{rpm}$ and at $10^{\circ} \mathrm{C}$; all samples were stored at $-20^{\circ} \mathrm{C}$ until analysis.

PGFM was assayed according to published methods ${ }^{1011}$ with validated modifications. ${ }^{12}$ Briefly, $3 \mathrm{ml}$ of plasma was extracted twice with a mixture of cyclohexane :ethyl acetate $(1: 1 \mathrm{v} / \mathrm{v})$ after acidification with citric acid. Further purification was achieved by applying samples dissolved in cyclohexane:ethyl acetate:methanol $(60: 40: 2$ by volume) to microcolumns containing silicic acid and eluting a prostaglandin fraction with cyclohexane:ethyl acetate:methanol (60:40:20 by volume). Fractions were evaporated under nitrogen pressure and resuspended in a phosphate buffer, $\mathrm{pH} 7 \cdot 4$. Samples were assayed in duplicate using an antiserum donated by $\mathrm{Dr} F$ Dray (Pasteur Institute, Paris). An iodinated ligand was used in the assay. ${ }^{11}$

Statistical analyses were performed using the Wilcoxon signed rank test for paired samples and the Mann-Whitney rank sum test for nonpaired samples.

\section{Results}

The mean concentrations of PGFM ( \pm SE of mean) in peripheral plasma before and five minutes after amniotomy and vaginal examination with and without membrane sweep are illustrated in the figure. Each procedure resulted in a significant increase $(P<0.01)$ in $P G F M$ concentrations. Differences in the mean concentrations of PGFM in the control samples from each group were not statistically significant $(\mathbf{P}>0.05)$. The levels of PGFM achieved after both amniotomy and vaginal examination with sweeping of the membranes were significantly greater $(P<0.05$ and $P<0.01$ respectively) than the levels after vaginal examination alone. The difference between the levels of PGFM after the former two procedures was not, however, significant. These

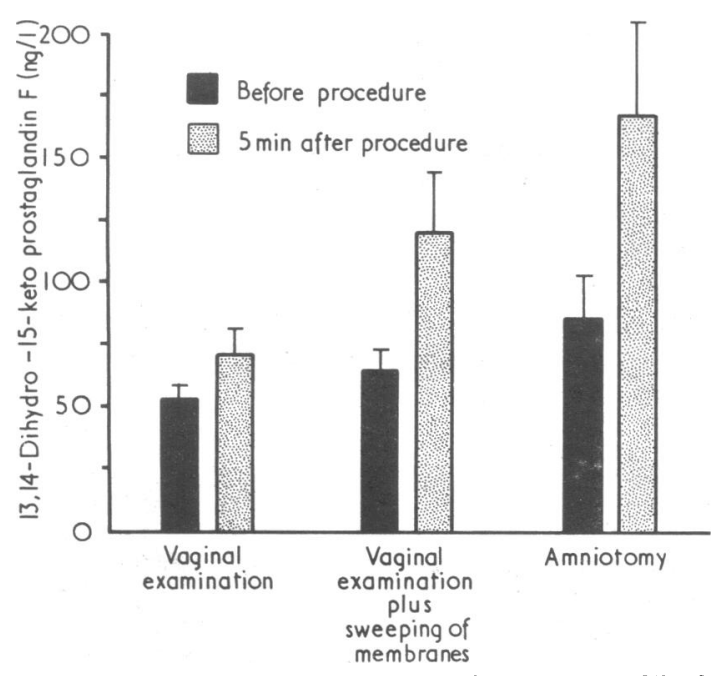

Peripheral plasma concentrations of PGFM (mean $\pm S E$ of mean) in women in late pregnancy before and five minutes after vaginal examination, vaginal examination plus sweeping of the membranes, and amniotomy. findings were equally true whether the data were analysed according to the absolute increase or the percentage increase in PGFM concentrations after each procedure.

\section{Discussion}

These results show that vaginal examination in late human pregnancy rapidly increases circulating prostaglandin concentrations. The fact that sweeping or rupturing the membranes increases the concentrations still further suggests that disruption of the membrane attachments to the uterine wall or the piercing of the membranes with leakage of amniotic fluid also provides a stimulus for prostaglandin release. The increased release of prostaglandins in response to amniotomy may therefore be the result of several components-namely, stimulation of the vagina and cervix and disturbance of the fetal membranes. Release of amniotic fluid at amniotomy with a consequent reduction in the volume of the uterus is unlikely to play a part, since the rise in prostaglandin concentrations was no different whether the membranes were punctured or not. This is consistent with previous data ${ }^{13}$ showing that labour was not induced by reducing the volume of amniotic fluid by amniocentesis in multiparous women with a favourable cervix at term, whereas amniotomy subsequently rapidly induced labour in the same women.

We preferred to measure the metabolite PGFM, for which there is now a sensitive and specific assay, rather than the primary prostaglandins since it circulates in higher concentrations $s^{1415}$ and is not produced by platelets ${ }^{16}$ or during storage. ${ }^{12}$ Hence it is the prostaglandin of choice to monitor in the peripheral circulation.

We measured these prostaglandin levels at only one time (five minutes) after the clinical examination. We chose this period because of the response in sheep to the same procedure, ${ }^{8}{ }^{9}$ but we do not know whether this is the time of maximal response because the relatively large volumes of plasma required for the assay deterred us from taking more than two blood samples from each patient. It therefore remains uncertain whether peak levels were reached at the same time in the three groups of patients studied. Since amniotomy is more successful in inducing labour than membrane sweep in late pregnancy, ${ }^{17}$ puncturing of the membranes may lead to a more sustained release of prostaglandins, possibly due to stimulation of the lower uterus and upper cervix by descent of the fetal presenting part, or by extension of the original puncture site on the membranes. There was no obvious relation between the degree of response to the clinical procedures and time to the onset of labour, although two patients in the amniotomy group who had abnormally high basal concentrations of PGFM did not require oxytocin and delivered rapidly after amniotomy.

Studies in sheep have indicated that oxytocin released via a neuroendocrine reflex in response to vaginal and cervical distension acts on the uterus to release PGF into the uteroovarian venous blood. ${ }^{818}$ Although cervical dilatation has been shown to increase uterine contractility in pregnant women near term, ${ }^{19}$ this effect was apparently not due to reflex oxytocin secretion. If oxytocin secretion is not stimulated by vaginal examination in pregnant women and since prostaglandin concentrations are raised in amniotic fluid within minutes of amniotomy, ${ }^{7}$ these findings strongly indicate a local stimulus for, and control of, prostaglandin secretion.

The source of the prostaglandins that can be secreted so rapidly in response to genital tract stimulation remains uncertain, although the decidua ${ }^{4}$ and fetal membranes ${ }^{5}$ are obvious possibilities. It is not, however, proved that this effect occurs only in pregnant women; indeed the release of prostaglandins during sexual intercourse may play a part in sperm transport, although it is seminal prostaglandins that are usually implicated in this process. ${ }^{20}$ In certain ethnic groups the belief is held that sexual intercourse during labour is helpful in accelerating labour and it may be that the release of prostaglandins is involved.

These findings have implications concerning, for example, 
vaginal examination in assessing preterm labour or the placement of a suture around the cervix in pregnancy. Both of these procedures may possibly release prostaglandins when increased uterine activity is not desirable. Our studies are being extended in these directions.

We thank Mrs H Hodgson and the staff of the John Radcliffe Hospital for their help with this study and Dr F Dray for the kind gift of antiserum. This work was supported by a grant from the Medical Research Council to ACT.

\section{References}

1 Thorburn, G D, Challis, J R G, and Currie, W B, Biology of Reproduction, 1977, 16, 18.

2 Challis, J R G, Robinson, J S, and Thorburn, G D, The Fetus and Birth, p 211. Amsterdam, Elsevier-Excerpta Medica-North Holland, 1977.

3 Turnbull, A C, et al, The Fetus and Birth, p 427. Amsterdam, ElsevierExcerpta Medica-North Holland, 1977.

' Gustavii, B, The Fetus and Birth, p 343. Amsterdam, Elsevier-Excerpta Medica-North Holland, 1977.

${ }^{5}$ MacDonald, P C, et al, Obstetrics and Gynecology, 1974, 44, 629.
${ }^{6}$ Keirse, M J N C, and Turnbull, A C, British fournal of Obstetrics and Gynaecology, 1976, 83, 146

${ }^{7}$ Mitchell, M D, et al, British fournal of Obstetrics and Gynaecology, 1975, $\mathbf{8 4}, 35$.

8 Flint, A P F, et al, fournal of Reproduction and Fertility, 1975, 43, 551.

${ }^{9}$ Mitchell, M D, Flint, A P F, and Turnbull, A C, fournal of Reproduction and Fertility, 1977, 49, 35.

10 Dray, F, Charbonnel, B, and Maclouf, J, European fournal of Clinical Investigation, 1975, 5, 311.

${ }_{11}$ Sors, H, et al, Biochemica et Biophysica Acta, 1977, 486, 553.

${ }^{12}$ Mitchell, M D, et al. Submitted for publication.

${ }^{13}$ Csapo, A I, et al, American fournal of Obstetrics and Gynecology, 1963, 85, 819

${ }^{14}$ Béguin, F, et al, Acta Physiologica Scandinavica, 1972, 86, 430

${ }^{15}$ Gréen, K, et al, American fournal of Obstetrics and Gynecology, 1974, 120, 25.

${ }^{16}$ Samuelsson, B, Les Prostaglandines, Seminaire Inserm, p 21. Paris, INSERM, 1973.

17 Swann, R O, Obstetrics and Gynecology, 1958, 11, 74.

${ }^{18}$ Mitchell, M D, Flint, A P F, and Turnbull, A C, Prostaglandins, 1975, 9,47 .

${ }^{19}$ Fisch, L, Sala, N L, and Schwarz, R L, American fournal of Obstetrics and Gynecology, 1964, 90, 108.

${ }^{20}$ Eliasson, R, Acta Physiologica Scandinavica, 1959, 158, suppl, p 1.

(Accepted 2 September 1977)

\title{
Association of serum lipids and obesity with cardiovascular mortality
}

\author{
RISTO PELKONEN， ESKO A NIKKILÄ，SEPPO KOSKINEN， KARI PENTTINEN, SEPPO SARNA
}

British Medical fournal, 1977, 2, 1185-1187

\section{Summary}

Serum lipid concentrations, relative body weight, and smoking habits were assessed in a cohort of 1648 middleaged Finnish men who were subsequently followed for seven years. Multivariate analysis showed that serum triglyceride and cholesterol concentrations and smoking were all independently associated with cardiovascular mortality. High serum triglyceride concentrations increased the risk of cardiovascular death only when they exceeded $1.7 \mathrm{mmol} / 1(150 \mathrm{mg} / 100 \mathrm{ml})$, but this occurred at all cholesterol and relative body weight levels. Obesity influenced the death rates only in men with raised serum lipid levels, while smoking was associated with increased mortality when any combination of the other factors was present. Men who had raised triglyceride concentrations combined with smoking or obesity had the highest risk of cardiovascular death.

\footnotetext{
Third Department of Medicine, University of Helsinki, Helsinki, Finland

RISTO PELKONEN, MD, consultant in endocrinology

ESKO A NIKKILÄ, MD, professor of medicine

Life Insurance Company Suomi-Salama, Helsinki, Finland

SEPPO KOSKINEN, MA, actuary

KARI PENTTINEN, MD, professor of virology

Department of Public Health Science, University of Helsinki, Helsinki, Finland
}

SEPPO SARNA, PHD, associate professor in biostatistics

\section{Introduction}

Several prospective studies have uniformly shown that ischaemic heart disease is closely associated with high serum cholesterol concentrations and blood pressure levels and with cigarette smoking. ${ }^{1-7}$ Raised serum triglyceride concentrations have also been connected with ischaemic heart disease, but evidence for their independent role has remained controversial. ${ }^{4} 87$ Familial hypertriglyceridaemia seems not to be associated with a high incidence of ischaemic heart disease ${ }^{8}$ : it is uncommon among patients with acute myocardial infarction and their relatives. ${ }^{\circ}$ Another factor whose status as a risk factor for ischaemic heart disease is still disputed is obesity. Some studies have shown a clear association between excessive body weight and an increased incidence of ischaemic heart disease,,$^{3510}$ but several others have not confirmed this. ${ }^{124611}$ We have investigated these problems further by conducting a seven-year prospective study in a cohort of middle-aged Finnish men. We tried to find an association between cardiovascular mortality and several supposed risk factors-serum triglyceride and cholesterol concentrations, relative body weight, and cigarette smoking-singly or in combination.

\section{Subjects and methods}

The study group was composed of men aged 50 to 53 years who had been insured with one large Finnish life insurance company (Suomi-Salama) for at least 10 years. In 1965 and 1966 all the men meeting these criteria were invited to participate in the study. Of a total of 5454 men, $39.7 \%$ responded and after those with incomplete data had been excluded a cohort of 1648 men remained.

At the start of the study each man was examined: his serum cholesterol $^{12}$ and triglyceride ${ }^{13}$ concentrations, height, and weight were measured and his smoking habits assessed. The serum lipids were analysed from a sample taken after a 12-hour overnight fast. The weight:height ratio $(\mathrm{kg} / \mathrm{m})$ was taken as a measure of the relative body weight. A smoker was defined as a person who smoked cigarettes 